\title{
Radial Carboxyterminal Peptides of Tubulin Directly Imaged in Microtubules by Electron Microscopy Tomography Exploiting Emplitude Contrast
}

\author{
Andrea Fera ( $\sim$ andrea.fera@gmail.com ) \\ National Institute of Child Health and Human Development \\ Thomas Reese \\ National Institute of Neurological Disorders and Stroke \\ Joshua Zimmerberg \\ National Institute of Child Health and Human Development \\ Dan Sackett
}

National Institute of Child Health and Human Development

\section{Research Article}

Keywords: Tubulin carboxyterminal tails, electron microscopy tomography, tungstate salts

Posted Date: April 30th, 2021

DOI: https://doi.org/10.21203/rs.3.rs-409249/v1

License: (a) (i) This work is licensed under a Creative Commons Attribution 4.0 International License.

Read Full License 


\title{
Radial carboxyterminal peptides of tubulin directly imaged in microtubules by electron microscopy tomography exploiting amplitude contrast.
}

\author{
Andrea Fera $^{* \neq}{ }^{\circ}$, Thomas S. Reese ${ }^{\wedge}$, Josh Zimmerberg ${ }^{*}$, and Dan L. Sackett ${ }^{\star}$ \\ * Division of Basic and Translational Biophysics, Eunice Kennedy Shriver National Institute of Child Health \\ and Human Development (NICHD), Bldg 9 RM 1E106 \\ $\ddagger$ Reveal Global Consulting LLC, 8115 Maple Lawn Blvd, Suite 375, Fulton, MD \\ $\wedge$ Laboratory of Neurobiology, National Institutes of Neurological Disorders and Stroke (NINDS), Building 49 \\ Room 3E13 \\ National Institutes of Health, 9000 Rockville Pike, 20892 Bethesda, MD \\ 'corresponding author: andrea.fera@gmail.com \\ Mail Address: Reveal Global Consulting LLC, 8115 Maple Lawn Blvd, Suite 375, Fulton, MD
}

Authors Contributions: AF did all electron microscopy experiments, data analysis, figure preparation, discussions of results/interpretation and wrote the text; TSR participated to the planning of experiments, discussed the analysis, and contributed to figures and text; JZ organized microscope and discussed the results; DLS did the tubulin preparation and optimization, the microtubule assembly on EM grids, the staining protocol, discussed all data elaboration and participated in all writing.

\section{Abstract}

Tubulin carboxyterminal tails (CTT) are peptides of 10-20 amino acids, unstructured and acidic, that vary in sequence between tubulin isotypes and are exposed on the outer surface of microtubules (MTs). These peptides have, so far, eluded direct visualization. In this report, electron microscopy tomography was applied to isolated MTs stained with Uranyl and tungstate salts demonstrated to resist sustained electron beam irradiation. Such resistance of high electron doses allows each electron microscopy image to be recorded with a high signalto-noise ratio. Corresponding tomograms reconstructed from tilt series at high magnification show exceptional resolution of details, revealing features of 
average dimension $\sim 1 \mathrm{~nm}$ without the need of averaging multiple samples. The known three-dimensional structure of the MT wall is apparent. But now images also reveal small stalks on the outer surface of MTs. Inspection of virtual sections demonstrates that the stalks are up to $\sim 2.5 \mathrm{~nm}$ long and $\sim 1 \mathrm{~nm}$ wide (at half length), protruding every $4 \pm 0.8(22) \mathrm{nm}$ along the microtubule. This spacing corresponds to one stalk per tubulin monomer. The grafting point on each monomer is not random but is positioned at one end of each monomer, identifying that end as toward the (-) end of the MT. The stalks are not observed following CTT removal with subtilisin. We conclude that these stalks are the CTT peptides of tubulin.

\section{Introduction}

It has long been possible to image individual molecular details by electron microscopy using staining protocols [1, 2]. Recently, a staining protocol has been combined with tomography in order to produce a three-dimensional representation of un-averaged biological samples at sub-nanometer level [3, 4]. The idea of using mainly amplitude contrast in order to generate images at high resolution with electron microscopy tomography (EMT) is not new and routinely used in material science [5-7]. Applications to biological samples have been less frequent [8], and often without pushing the technique to observations in threedimensions at the resolution afforded by the data [9]. It may be important to note 
here that the recent results from negative stained samples demonstrated experimentally $[3,4,10]$ that the well-known estimation of many years ago for the resolution intrinsic to negative staining proteins [1] was intended as a point of reference for a new technique whose boundaries were yet uncertain. Indeed that estimation is not supported by measurements on actual calibrated samples. At the time the understanding of the formation of metallo-organic interactions, which is at the basis of the staining process in electron microscopy (EM), was rather new. More recent chemistry studies of interactions between proteins and transition metal ions $[11,12]$ demonstrate the strength and specificity of such interactions, which explain why the metal coats tightly the proteins upon evaporation of the solvent, that always precedes observation with an electron microscope. These studies underline, for instance, the reason why common closed-shell salts (like $\mathrm{NaCl}$ ) cannot be used as biological stains. In summary, most recent electron microscopy results implicitly demonstrate that specific negative stains chemically bind to the surface of proteins, maintaining their position during a tomography experiment. Therefore implying also, in turn, that the hypothesized disintegration of the organic material upon sustained irradiation with high-energy electron beams [13] may happen without observable rearrangements of the stain at length scales bigger than $\sim 0.3 \mathrm{~nm}$. In fact, these experiments are only sensitive to the position of the metal ions. In summary, in view of these strong coordination forces, metal ions report faithfully the waterexcluded surface of proteins [14]. 
Other sample preparation methods allow better conservation of molecular characteristics for EM imaging, at the expense of averaging data from multiple samples in order to achieve sufficient signal-to-noise ratio $(\mathrm{S} / \mathrm{N})$. Results are then shown employing various mathematical tools and, often, postulating specific molecular symmetry in the data $[15,16]$. In the material science field, when using EMT with amplitude contrast, like in the present work, it is fairly common to image molecular structures in three-dimensions with a resolution smaller than one nanometer, and without averaging multiple samples $[7,17]$.

Microtubules are rigid cytoplasmic polymers assembled from the heterodimer tubulin. The tubulin heterodimer consists of two very similar but not identical protein monomers, $\alpha-$ and $\beta$-tubulin. Both subunits have a globular portion containing $\sim 95 \%$ of the mass and a short (10-20 amino-acid residue) carboxy-terminal 'tail' peptide (CTT). The CTTs are acidic, unstructured, flexible, post-translationally modified, and protrude from each monomer at the outer surface of microtubules [18-22]. The CTTs protrude from a grafting site situated at one end of each monomer, toward the (-)-end of the microtubule [15]. These CTTs are 10-20 amino acid residues in length and rich in glutamic acid, corresponding to a maximal average length of $\sim 4-5 \mathrm{~nm}$ and an average diameter of $\sim 1 \mathrm{~nm}$ [20]. These characteristics make CTTs very challenging to image and indeed CTTs have never been directly imaged in their entirety. The properties of the CTTs have been studied also by molecular dynamics [20,21], but imaging methods have not revealed these flexible, heterogeneous peptide tails, maybe because other EM imaging methods rely on an increase of $\mathrm{S} / \mathrm{N}$ by averaging 
multiple samples. Lately, with an elegant preparation [23], it has been possible, using conventional cryo-tomography without contrasting agents, to resolve the portion of CTTs directly attached to the globular portion of tubulin. Nevertheless, the CTTs are not revealed in the high resolution images of MT-tau complexes [24]. Yet, resolving such radial peptides appears important since CTTs constitute a necessary first layer of interaction for molecules that approach the outer surface of a microtubule [22].

In this report it is demonstrated that it is possible, exploiting amplitude contrast, to image the CTTs of individual microtubules, using conventional transmission electron microscopy tomography, without the need for averaging multiple molecules. Here we report for the first time the presence of stalks approximately $1 \mathrm{~nm}$ in diameter and up to $\sim 2.5 \mathrm{~nm}$ long, protruding from each tubulin monomer of a microtubule. Their linear spacing along the microtubule protofilaments, their asymmetric grafting site on each monomer, and the demonstration of their sensitivity to known enzymatic cleavage, allows us to conclude that the stalks are the CTT peptides of tubulin.

\section{Results}

Microtubules (MTs) assembled from purified rat brain tubulin were stained with a modified version of the electron beam-stable stain previously described [3, 
4]. The expected morphology of MTs was found when MTs stained by this method were imaged by high magnification electron tomography (Figs 1,2 and 3).

First, we verified that these samples are not modified by exposure to the electron beam in vacuum during the collection of the 143 images necessary for this implementation of tomography (Figure 1a and b). Each image was obtained using $\sim 5000 \mathrm{e}^{-} / \mathrm{nm}^{2}$, for a total electron dose of about 1.5 millions $\mathrm{e}^{-}$during a tomography experiment. No changes were observed between the en-face image at the beginning (a) and at the end (b) of the experiment. No pixels in the images before the experiment were obscured or modified by sustained irradiation. In fact, a pixel-by-pixel subtraction of the two images gives a two-dimensional array with a null value of each pixel, within experimental errors $(3 \%)$. This experiment has been routinely repeated and carefully documented at both 200 and $120 \mathrm{keV}$ (Material and Methods section): the pixel-by-pixel subtraction gives a value corresponding to 1 to $3 \%$ of the electrons generating the signal in each pixel. All the fields of view of grids displayed this behavior. The stability of the samples was confirmed before/after each tomography experiment. It is this stability which allows the three-dimensional resolution discussed in the following.

Corresponding tomograms were reconstructed from 143 images throughout the range of accessible tilt angles, $-71^{\circ}$ to $+71^{\circ}$, as specified in the material and methods section. The virtual sections and three-dimensional rendering shown here correspond to one area chosen as representative. The complete field of view before and after the experiment is reported in Supplementary Figure 1. In Fig. 2 virtual sections (at inverted contrast), one voxel thick $(0.39 \mathrm{~nm})$, are shown along 
planes parallel to the supporting film (chosen as XY plane) on which the MT rested (panel 1). Planes are shown at increasing $Z$ values moving upwards until the hollow center of microtubule (panel 6). The thickness of such virtual sections is defined by the pixel size of the initial images, $0.39 \mathrm{~nm}$. As expected, individual protofilaments sequentially appear in successive sections, starting with the central (contact) protofilaments at the grid surface and adding new ones at both sides while stepping upwards through the microtubule. The bottom half of the microtubule, adjacent to the supporting film, was less flattened during drying than the top portion (Fig 4).

Individual tubulin monomers can be clearly distinguished as elements of protofilaments (Fig. 2b). The monomer repeat spacing along the protofilament axis is $4.0 \pm 0.2 \mathrm{~nm}$, and neighboring monomers in adjacent protofilaments display a shallow helical rise, as expected. In Fig $2 b$ the apparent value of the helical rise angle varies between $\sim 9.5^{\circ}$ and $\sim 14^{\circ}$, and protofilament lateral spacing varies between $\sim 4.2 \mathrm{~nm}$ and $\sim 5.7 \mathrm{~nm}$. These values are consistent with the expected values of $\sim 10.5^{\circ}$ for helical rise angle and $4.8 \mathrm{~nm}$ for protofilament spacing (average of exterior and interior faces), for a B-lattice, 13 protofilament microtubule [26] . Some variation from these values may be due to uncontrollable local drying conditions.

Tomograms allowed a three-dimensional image of a microtubule to be derived using automatic segmentation, after imposing a uniform threshold (Figure 3 ) to the reconstruction volume. A correction for the variation of the thickness of the scattering slab during the experiment (see methods) was implemented prior to 
reconstruction, to account for these specific experimental conditions, involving a $25 \mathrm{~nm}^{3}$ volume of stain, of thickness varying with the angle, which has to be penetrated by the radiation before arriving to the detector.

Results show that individual microtubules are not round but somewhat collapsed vertically, as expected and previously observed [26] showing a cross sectional axial ratio of $\sim 1.5$ (Fig 4). Microtubules collapsed to various degrees were typically found, and the extent of collapse appeared to depend on polymerization details or uncontrollable drying conditions.

The outer surface of this microtubule clearly shows a complex topography consisting of periodic protuberances. Stalks regularly protrude from each monomer along a protofilament (red circles in Fig 3) at a spacing of $4.0 \pm 1$ (22) $\mathrm{nm}$, which corresponds to the monomer spacing along the long protofilament axis. The stalks protrude from each monomer and are between 1 and $2.5 \mathrm{~nm}$ long, with a cross-sectional diameter (at half length) approaching $1.2 \mathrm{~nm}$. Notably, the stalks extend from one end of each monomer, all toward the same end of the MT (to the right in Fig 3), not from the monomer center nor randomly positioned within the monomer surface. These stalks can also be clearly seen in cross sections perpendicular to the long axis of the microtubule (Fig 4). Stalks are most evident (asterisks) on the lateral protofilaments, where the $\mathrm{S} / \mathrm{N}$ is maximal [26]. These stalks have the size, periodicity, and consistent asymmetric positioning on the monomer surface that is expected for the carboxyl-terminal tail (CTT) peptides of tubulin, known to be displayed on the outer surface of microtubules $[15,18,20,21$, 25]. 
This hypothesis has been tested by examining microtubules whose Ctermini have been cleaved enzymatically with subtilisin ("tubulin-S") $[18,25]$ by overnight cleavage. Controls were taxol-stabilized microtubules incubated overnight without added subtilisin. Comparison between cross-sectional virtual sections from intact and cleaved microtubules demonstrates that after cleavage with subtilisin the stalks disappear (Data not shown). In Fig 5 we report the most lateral protofilaments corresponding to a normal microtubule (left) and a subtilisindigested microtubule (right). This picture demonstrates that in the corresponding portion of a microtubule digested with subtilisin there are no stalks radially departing from the outer surface. Figure 5 also clearly illustrates the periodicity of the filaments on the undigested microtubule showing that these filaments are characteristic of the specific geometry of the microtubule, and not related to instrumental or technique limitations (which would produce a stochastic feature).

\section{Discussion}

Heavy metal staining is a well-known way to image biological specimens by EM [1, 26, 28-31], with limitations arising from the possibility of stain-induced artifacts. One of the most common stains is Uranyl acetate (UA). In recent years $[11,12,32,33]$ Uranyl chemical structure and binding properties have been better understood with respect to its interactions with organic molecules. Distortions provoked by negative staining must take into account the drying forces present at the sub-nanometer scale. Nevertheless, all stain artifacts seem likely confined to 
length-scales below the images discussed here [11, 12, 32]. Uranyl-based stains in excess have been demonstrated unstable under sustained electron irradiation [4]. However, a comprehensive assessment of the conditions in which a specific stain can resist irradiation is lacking. The discovery of a more beam-stable tungstate salt has been incorporated into the design of this study [3, 4]. This work demonstrates that not all Uranyl containing stains are unstable under sustained electron irradiation. A possible explanation of this new observation is that only metal ions not directly chemically coordinated by the organic material can be rearranged by sustained irradiation, as previously shown in [4]. Such rearrangements have not been observed here, possibly because the sample has been exposed twice to a solution of methylamine tungstate after UA (wicking excess solution each time, see methods), which may have eliminated the very soluble Uranyl ions not directly coordinated by the organic molecules.

Regardless the underlying mechanisms, beam-stable stains allow to take advantage of amplitude contrast to examine biological specimens, therefore unleashing the full resolution power of electron microscopes, as common in the material science field. Such resistance to dose is verified by many material science samples [7, 17], therefore justifying why it is routine in that field to image at the sub-nanometer level with non-aberration corrected microscopes. With enough amplitude contrast there is no need to average data from many samples, and therefore there is the possibility to image unique events like molecular reaction mechanisms or dislocations in crystals. 
Here we achieve sufficient three-dimensional resolution to resolve a map of dimensions corresponding to one protein, with enough resolution to distinguish a single extended peptide of diameter $\sim 1 \mathrm{~nm}$, using conventional non-aberration corrected electron microscopes, operated at $200 \mathrm{keV}$ (when available) or $120 \mathrm{keV}$ (routinely). We refer the reader to Material and Methods for further discussion on resolution. Stain stability has also been verified at $100 \mathrm{keV}$ and $300 \mathrm{keV}$ (data not shown). This result shows that amplitude contrast can be used also to image biological samples at high magnification with high $\mathrm{S} / \mathrm{N}$, yielding three-dimensional electron density maps with voxel sizes in the Angstrom range, and without averaging many (thousands of) samples [5].

A significant experimental finding underlying this study is that stain reorganization was not observed in these experimental conditions, regardless of the actual mechanism. A careful assessment of sample invariance upon doses complementary to a tomography experiment (cfr. Fig S2 at $120 \mathrm{keV}$ ) guaranteed that observations were not corrupted by rearrangements of metal salt, previously only observed in excess of UA [4]. Such observations later allowed to combine this method with cryo-fixation protocols [10], which combination seems necessary for ruling-out possible distortions of proteins intrinsic to air-drying.

Here EMT was exploited to reveal the unstructured flexible peptides, or CTT, that extend from the microtubule surface. Such details are averaged-away using EM imaging methods that require combination of images from identical particles $[1,2,15,30,34]$, which is a necessary procedure to obtain enough $\mathrm{S} / \mathrm{N}$ in absence of contrasting agents like transition metal salts. With staining, the 
increasing thickness of the layer containing high scattering atoms in the path of the radiation at increasing angles had to be corrected for (see Materials and Methods); after this correction was it possible to apply a single, uniform isodensity threshold to segment an entire area and render/examine sub-tomograms (Figures 2, 3, 4 and 5).

Stalks that were imaged appear regularly, with a spacing identical to the monomers to which they are grafted. Significantly, the grafting site is always eccentric, at one end of each monomer, and at the same end for all. From the known position of the grafting site [15] toward the (-) end of the microtubule, and the $\alpha$-end of the dimer, it is possible to identify the $\alpha$ and $\beta$ ends of the dimer. Only about half or less of the length of the CTT are visualized: $1-2.5 \mathrm{~nm}$ out of $\sim 4 \mathrm{~nm}$ [20], possibly also because it was still somewhat arbitrary, in this preparation, where to fix the threshold for rendering. Yet, it is noteworthy that the same threshold has been used for all details in the three-dimensional rendering shown (Figure 3).

Tubulin CTTs regulate interactions of microtubules with other proteins. We can now image these structures on single microtubules without averaging. The microtubules in this study were prepared from purified rat brain tubulin, which allows experimental manipulation of the sample, such as preparing microtubules with the CTTs cleaved. Therefore this work opens the way to investigate directly interactions of microtubules with MAPs or virus upon changing conditions as induced by drugs. 
The top portion of these microtubules appears more flattened than the bottom, suggesting that inter-protofilament connections are less strong than the surface tension of an evaporating water front in air $\left(\sim 70 \mathrm{dyn} / \mathrm{cm}^{2}\right)$. Such reference value should be measured in the conditions of the experiment, but it may be of further utility for molecular dynamics simulations. This has also been observed when microtubules are dried in air on mica, prior to atomic force microscopy, in which case the flattening is more extreme [35].

Limitations of this approach are the possible deformations of proteins resulting from interactions with heavy metal ions, intrinsic to the staining process. Nevertheless, in the present understanding of the specificity, strength and directionality of these interactions [11,32] it seems unlikey that molecular deformations occur at length scales much bigger than twice the size of coordinating heavy metal ions, whose average dimensions is less than $2 \AA^{3}$. Metal ions seem to coat the proteins quite nonspecifically, possibly following formation of this bond upon evaporation of the solvent (water), that always precedes insertion in the electron microscope. Actually, cylindrical protein samples have been reported compressed at the nanometer level $[1,16,29]$, but three-dimensional maps of the electron density (and with different stains) are not available, so that it is unclear if the coordination or drying forces are the driving factor for the observed distortions in all these cases, including the ones reported in this work. Further experiment with protocols similar to [10] could help to shed light on this point. It may be notable that microtubules collapse much more, nearly to flatness, when 
dried in air without stain and imaged with atomic force microscopy [35], suggesting that the surrounding stain may somewhat stabilize the microtubule wall.

Neighboring dimers in the same rendered protofilament often do not appear identical, although at these high magnifications the role of stain during drying is unclear (Fig. 2). It is known that there is a range of possible drying and local effects to consider $[2,28,30]$. This presents challenges to interpretation and opportunities for sample improvement, such as in a recently proposed protocol [10] .

In summary, this work demonstrates that single peptide tails on tubulin can be observed on the outer surface of microtubules. This is made possible by appropriate staining which allows resolution of single, unstructured peptides by EM tomography without the need to average between different samples.

\section{Materials and Methods}

Brain tubulin was purchased from Cytoskeleton (Denver $\mathrm{CO}$ ) or was isolated from rat brain as described previously [18] and stored in liquid nitrogen until use. No rats were ever housed or sacrificed in our laboratory and this study is not subject to institutional ethical review since no animals were ever directly involved. All experiments here utilized PM buffer: $0.1 \mathrm{M}$ Pipes, $1 \mathrm{mM} \mathrm{MgCl}$, pH 7.0. Assembly of tubulin to Taxol-stabilized microtubules was performed by supplementing a 10 $\mu \mathrm{M}$ tubulin solution in PM buffer with $1 \mathrm{mM}$ GTP followed by $4 \mu \mathrm{M}$ Taxol, then an additional $16 \mu \mathrm{M} \mathrm{Taxol}$, with 5 minutes at $37^{\circ} \mathrm{C}$ in between each addition. Samples were then incubated at room temperature for 45 minutes. Assembly of tubulin to GMPCPP-stabilized microtubules was performed by supplementing a $10 \mu \mathrm{M}$ tubulin solution in PM buffer with $0.5 \mathrm{mM}$ GMPCPP and incubating at $37^{\circ} \mathrm{C}$ for 30 minutes. Subtilisin digestion was performed according to $[18,25]$. 
Microtubules were polymerized in presence of guanosine-5'-[( $\alpha, \beta)$ methyleno]triphosphate (GMPCPP) or Taxol, as indicated, before deposition on 200-square mesh copper grids, covered with carbon-only or formvar-carbon films. Grids were floated on $\sim 10 \mu$ solution containing $1 \mathrm{mg} / \mathrm{ml}$ of microtubules. The excess was wicked away after $\sim 20$ seconds.

An intermediate step was performed before negative staining the microtubules with Methylamine Tungstate (NanoW, Nanoprobe), used as supplied ( $2 \% \mathrm{w} / \mathrm{v}$ at $\mathrm{pH}$ 6.8). Microtubule stabilization was first performed by applying Uranyl Acetate (UA) as described below, resulting in stable samples under the sustained irradiation intrinsic to this imaging protocol. All results shown here have been obtained on stabilized microtubules fixed with UA before further negative staining, as described below.

Staining protocol: $\sim 10 \mu \mathrm{l}$ drop of UA solution $1 \% \mathrm{w} / \mathrm{v}$ was left in contact with freshly deposited microtubules wicking the excess after $\sim 10$ seconds. Successively the grid was washed with distilled water very quickly and negative stained with NanoW. Alternatively, application of NanoW was done with two drops, the first one left in contact with the sample for $\sim 3$ seconds, wicking excess, and floating grid on the second one for $\sim 1$ minute. Excess was wicked off again before drying in air for at least 2 minutes, before storing the grids vertically prior to microscopy. Imaging was realized usually within one week from the sample deposition.

Fiduciary markers: depending on the magnification used, 3 , or $5 \mathrm{~nm}$ gold beads were chosen. In Supplementary Figure 1 colloidal $5 \mathrm{~nm}$ gold spheres were used as fiduciary markers. They were deposited on the surface and allowed to dry before depositing the microtubules, as described above.

Microtubules imaged after digestion with subtilisin were first stabilized with Taxol and then digested overnight before staining as described. Controls were Taxol microtubules left overnight at $37^{\circ} \mathrm{C}$ in incubator without subtilisin. 


\section{Electron Microscopy Tomography}

Experiments were carried out with a Tecnai TEM electron microscope operating at $200 \mathrm{keV}$ when available, otherwise routinely with a Jeol TEM electron microscopes operating at $120 \mathrm{keV}$. Microscopes were equipped with a LaB6 source. No differences regarding rearrangement of stain under irradiation were observed operating routinely at all acceleration voltages listed above and at a magnification leading directly a pixel size from $\sim 1$ to $0.21 \mathrm{~nm}$. Stability was tested until $100 \mathrm{keV}$ acceleration voltages, and stain rearrangements were never observed. We speculate that this behavior is a consequence of the fact that tungstate ions are in a stable closed-shell configuration, and the Uranyl ions remained on the sample are stably chemically coordinated by the numerous Lewis-bases on the surface of proteins [11,32]. Detectors were routinely operated in 'counting mode' for all experiments, i.e. actual counts recorded in each pixel were directly proportional to the number of electrons generating the signal. Poisson statistics could therefore be used to assess accuracy of each voxel. This characteristic of the signal has not been fully exploited in this work, although it facilitates exams of direct images and of reconstructed volumes, when the error propagation is done correctly.

Microtubules digested with subtilisin were imaged at $120 \mathrm{keV}$ with a Jeol microscope operated with a LaB6 source. After verification as described, images were obtained at a magnification leading directly a pixel size of $0.21 \mathrm{~nm}$ or higher. The other microtubules here reported were stabilized with GMPCPP and imaged at $200 \mathrm{keV}$, at a magnification leading directly a pixel size of $0.39 \mathrm{~nm}$. No differences have been noted for the purposes of this work between Taxol- and GMCPP-stabilized microtubules.

In order to achieve high-enough resolution the experimental geometry chosen was uni-axial (as usual in material science experiments), with $1^{\circ}$ angular step between images, for a total of 143 images in each experiment [3,4], in order to satisfy the criterion of enough information content in a tomogram to achieve the desired resolution [27]. The objective aperture of the electron microscopes was either removed or larger than $100 \mu \mathrm{m}$ diameter in all experiments here reported. Further 
experiments are underway to assess the actual limit for accessing information of molecular dimensions. The most stringent limitation here seems to be the resolving power of the microscope used, that depends on the wavelength of the radiation (120 or $200 \mathrm{keV}$ ) with this contrast method.

The explicit choice done in this work has been to use mainly amplitude scattering (i.e. the real part of the scattering wave function) as the source of image contrast. This choice enables us to use the straight approach (introduced by Abbe and Rayleigh in the last two centuries) for calculating image resolution, provided that enough signal-to-noise $(\mathrm{S} / \mathrm{N})$ distinguishes values measured by two neighboring pixels in the image.

For all practical purposes in optical microscopy this translates in considering the theoretical resolution as half the wavelength of the radiation. Since in these experiments the wavelength of accelerated electrons is a few picometers, the theoretical resolution of an electron microscope accelerating the electrons to 200 $\mathrm{keV}$ is only limited by electro-optical aberrations to approximately $0.2 \mathrm{~nm}$ [36]. The calculated virtual sections shown here would have actually an increased resolution with respect to the initial images, acquired at $0.37 \mathrm{~nm}$ pixel size. This increase is thought to be inversely proportional to the number of images combined in order to calculate the virtual sections in the tomogram [27]. Considering the grain size of the stain, images have been acquired around the tabulated power of resolution of the microscope used. The pixel size chosen was $0.37 \mathrm{~nm}$ (at $200 \mathrm{keV}$ ) or $\sim 0.25 \mathrm{~nm}$ (at $120 \mathrm{keV}$ ). The reader should consider that, in the conditions of the experiment, a small amount of electrons may be scattered at angles such that the signal will be recorded in the pixels neighboring where the scattering phenomenon occurred.

Considering these factors, but because we recorded the images using an electron dose allowing to distinguish the intensity in adjacent pixels (since the EM signal is acquired in Poissonian statistics), we think reasonable to conclude that all images reported in this article, and therefore also all corresponding renderings, have a resolution of at least two pixel sizes, i.e. $\sim 0.7 \mathrm{~nm}$. This result has been obtained from one experiment done in one day, with the successive 
elaboration done in a few hours, after optimizing the sample preparation protocol and the microscope alignment.

Experiments were conducted using a high-tilt Gatan holder bi-axial, although a mono-axial acquisition protocol was chosen. Experiments were routinely set between $\pm 71^{\circ}$, recording an image every $1^{\circ}$ of rotation, setting the acquisition software (SERIALEM) for counting at least $5000 \mathrm{e}-/ \mathrm{nm}^{2}$ per image. Therefore the total electron dose intrinsic to an experiment was of the order of $10^{6} \mathrm{e} / \mathrm{nm}^{2}$, since the beam was not obscured during movements of the stage.

Raw images as function of angle between the electron beam and the sample have been aligned using cross correlation spatial procedures and fiduciary markers of appropriate size. Fiduciary markers diameter was chosen to be roughly 15 pixels diameter at least.

Aligning of the raw images and centering has been implemented using the ETOMO/IMOD shareware package [37] of the university of Boulder, (CO). This packet has been used with all default parameters, besides indicating that fiduciary markers were only on one side during their alignment. Before the calculation of the tomogram the aligned image stack from ETOMO has been corrected with the routine DENSNORM (included in the package). Such correction takes into account the extra weakening of the signal at low surface angles in view of the thick slab of stained material which the radiation has to penetrate before reaching the detector. Instead, it still has to be implemented a precise calculation of the error bars at the highest values of height $(z)$ in the reconstructed volume, which depends on the fact that a higher number of projections are used for calculating the most external voxels of the microtubules (in our specific application). This fact generated the choice of focusing on the information contained in the most external protofilaments of two microtubules in Fig. 5, in order to put in evidence the effect of Subtilisin only on one microtubule.

After aligning the raw images, the ETOMO package was also used to calculate corresponding tomograms both with the back-projection method and with SIRT. No major differences between virtual sections arising from these two methods of 
calculation have been observed. Nevertheless, when not otherwise specified, all results shown come from 30 iterations using SIRT.

Tomogram output has been routinely polished using Gaussian blur spatial

filter of Full-Width-Half-Maximum (FWHM) $\sim 0.8$ up to $\sim 1.5$ pixels. This choice has

been dictated by the need of not inserting unwanted information in the tomogram

of the order of a few pixel sizes. Rendering of tomograms has been realized

imposing a constant threshold to the whole dataset displayed with Chimera

(shareware software from UCSF).

\section{Bibliography}

[1] M. Ohi, Y. Li, Y. Cheng, T. Walz, Biol. Proced. Online, 6 (2004) 23-34.

[2] C.H. A Bremer, A Engel, W Baumeister and U Aebi, Ultramicroscopy, 46 (1992) 85-111.

[3] A. Fera, A. Dosemeci, A.A. Sousa, C. Yang, R.D. Leapman, T.S. Reese, J Comp Neurol, 520 (2012) 4218-4225.

[4] A. Fera, J.E. Farrington, J. Zimmerberg, T.S. Reese, Microsc Microanal, 18 (2012) 331-335.

[5] L. Reimer, H. Kohl, Transmission Electron Microscopy - Physics of Image Formation 5ed., Springer 2008.

[6] L. Reimer, M. Ross-Messemer, Journal of Microscopy, 155 (1988) 169-182.

[7] M.C. Scott, C.C. Chen, M. Mecklenburg, C. Zhu, R. Xu, P. Ercius, U. Dahmen, B.C. Regan, J.W. Miao, Nature, 483 (2012) 444-447.

[8] R.W.H. Ruigrok, N.G. Wrigley, L.J. Calder, S. Cusack, S.A. Wharton, E.B. Brown, J.J. Skehel, EMBO J, 5 (1986) 41-49.

[9] J. Mast, L. Demeestere, Diagnostic Pathology, 4 (2009) 5.

[10] A. Fera, L. Dye, Microscopy and Microanalysis, 23 S1 (2017) 1114.

[11] G. Brancatelli, S.G., A. Notti, A. Pappalardo, G. Trusso Sfrazzetto, Mono and dinuclear uranyl(VI)

complexes with chiral Schiff base ligand. Inorg. Chimica Acta, 2013. 396: p. 25-29.

[12] K.L. Haas, K.J. Franz, Chemical Reviews, 109 (2009) 4921-4960.

[13] S. Tavernier, Experimental Techniques in Nuclear and Particle Physics, Springer2010.

[14] R. Adamczak, A. Porollo, J. Meller, Proteins, 59 (2005) 467-475.

[15] G. Borisy, R. Heald, J. Howard, C. Janke, A. Musacchio, E. Nogales, Nat Rev Mol Cell Biol, 17 (2016) 322-328; Nogales E, Wolf SG, Downing KH, Nature. 1998 Jan 8;391(6663):199-203

[16] R.H. Wade, D. Chretien, Journal of Structural Biology, 110 (1993) 1-27.

[17] X. Ye, M.R. Jones, L.B. Frechette, Q. Chen, A.S. Powers, P. Ercius, G. Dunn, G.M. Rotskoff, S.C. Nguyen, V.P. Adiga, A. Zettl, E. Rabani, P.L. Geissler, A.P. Alivisatos, Science, 354 (2016) 874-877.

[18] Sackett, D.L., B. Bhattacharyya, and J. Wolff, Tubulin Subunit Carboxyl Termini Determine Polymerization Efficiency. Journal of Biological Chemistry, 1985. 260(1): p. 43-45.

[19] J. Lefevre, K.G. Chernov, V. Joshi, S. Delga, F. Toma, D. Pastre, P.A. Curmi, P. Savarin, J Biol Chem, 286 (2011) 3065-3078. 
[20] H. Freedman, T. Luchko, R.F. Luduena, J.A. Tuszynski, Proteins, 79 (2011) 2968-2982.

[21] K.P. Wall, M. Pagratis, G. Armstrong, J.L. Balsbaugh, E. Verbeke, C.G. Pearson, L.E. Hough, Acs Chemical Biology, 11 (2016) 2981-2990.

[22] K.T. Wallis, S. Azhar, M.B. Rho, S.A. Lewis, N.J. Cowan, D.B. Murphy, Journal of Biological Chemistry, 268 (1993) 15158-15167.

[23] C.P. Garnham, A. Vemu, E.M. Wilson-Kubalek, I. Yu, A. Szyk, G.C. Lander, R.A. Milligan, A. RollMecak, Cell, 161 (2015) 1112.

[24] Elizabeth H. Kellogg, Nisreen M. A. Hejab, Simon Poepsel, Kenneth H. Downing, Frank DiMaio, Eva Nogales, Science. 2018 Jun 15;360(6394):1242-1246. doi: 10.1126/science.aat1780. Epub 2018 May

10.PMID: 29748322

[25] D.L. Sackett, J. Wolff, Journal of Biological Chemistry, 261 (1986) 9070-9076.

[26] E.M. Mandelkow, R. Schultheiss, R. Rapp, M. Muller, E. Mandelkow, J Cell Biol, 102 (1986) 10671073.

[27] D.J. De Rosier, A. Klug, Nature, 217 (1968) 130-134.

[28] J.R. Harris, R.W. Horne, Micron, 25 (1994) 5-13.

[29] N.A. Kiselev, M.B. Sherman, V.L. Tsuprun, Electron Microscopy Reviews, 3 (1990) 43-72.

[30] R.M. Oliver, Meth Enzym, 27 (1973) 616-672.

[31] S.A. Burgess, M.L. Walker, K. Thirumurugan, J. Trinick, P.J. Knight, Journal of Structural Biology, 147 (2004) 247-258.

[32] F.P. Ballistreri, G.L., A. Pappalardo, F. Punzo, A. Thompson, G.A. Tomaselli, R.M. Toscano, G.

Trusso Sfrazzetto, An integrated X-ray and molecular dynamics study of uranyl-salen structures and properties. Dalton Trans, 2012. 41: p. 1951-1960.

[33] J. Howatson, D.M. Grev, J Inorg Nucl Chem, 37 (1975) 1933-1935.

[34] S. Banerjee, A. Bartesaghi, A. Merk, P. Rao, S.L. Bulfer, Y. Yan, N. Green, B. Mroczkowski, R.J. Neitz, P. Wipf, V. Falconieri, R.J. Deshaies, J.L.S. Milne, D. Huryn, M. Arkin, S. Subramaniam, Science, 351 (2016) 871-875.

[35] Hamon, Loic \& Curmi, Patrick \& Pastre, David. (2010). Methods in cell biology. 95. 157-74. DOI

10.1016/S0091-679X(10)95009-7.

[36] David Smith, Ultimate Resolution in the Electron Microscope?, Materials Today - Microscopy ; Special Issue, pp 30-38, ISSN:1369 7021 @ Elsevier Ltd 2008

[37] Kremer J.R., D.N.M.a.J.R.M., Computer visualization of three-dimensional image data using IMOD. J. Struct. Biol, 1996. 116: p. 71-76. 


\section{Figures}

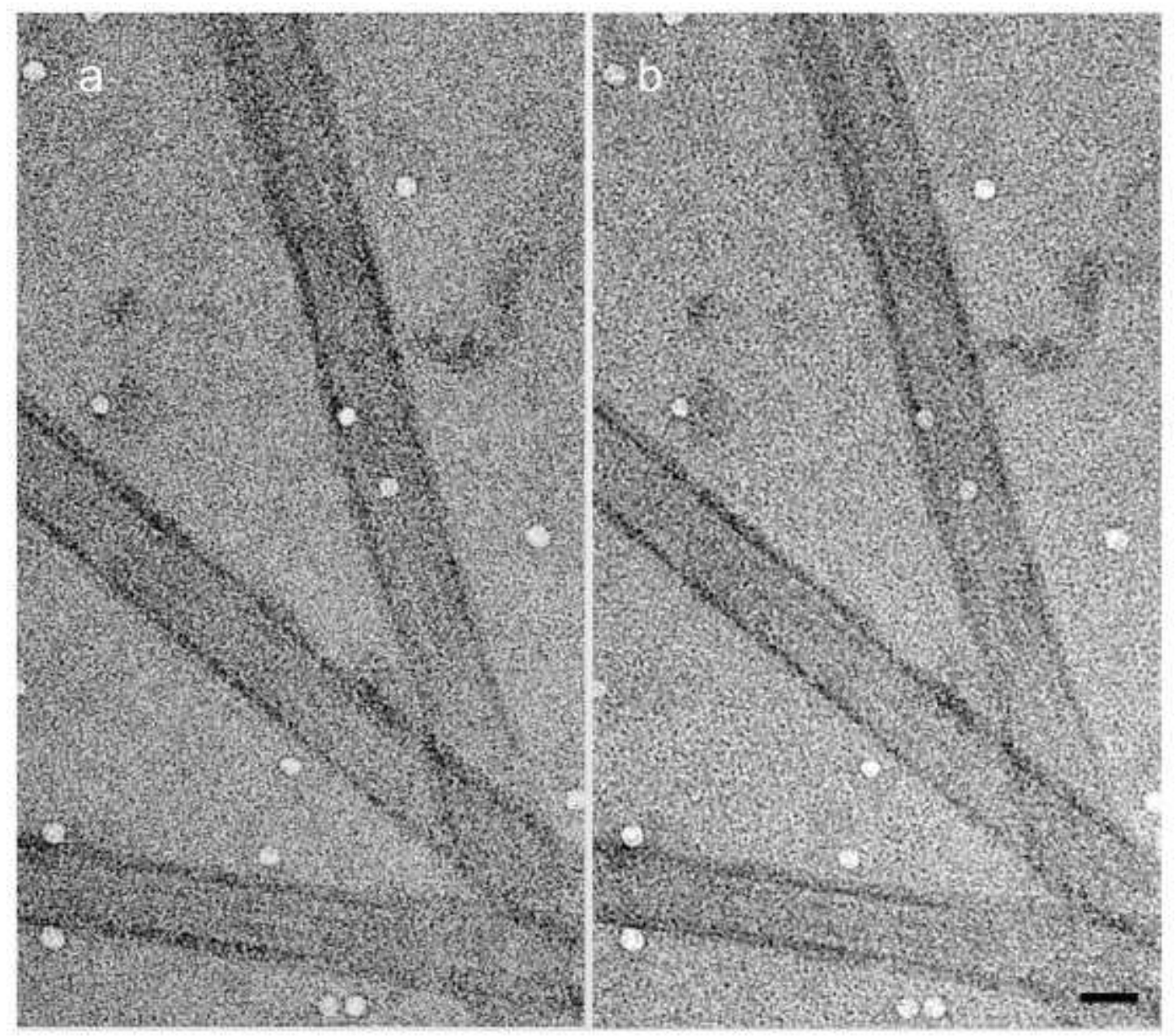

\section{Figure 1}

Microtubules stabilized and negative stained imaged en-face at $200 \mathrm{keV}$. A representative portion of the $4000 \times 4000$ pixel field of view of the camera is shown; whole field of view available in Supplementary Material. The contrast has been inverted. Images were acquired just before (a) and immediately after (b) collection of a tomography series of 143 images. No features appear to vary during the experiment. The colloidal gold in the picture has a nominal diameter of $5 \mathrm{~nm}$. The two pictures were acquired with a Tecnai TF20 operated at $200 \mathrm{keV}$. Images were acquired in counting mode on a Gatan K2 Detector, at a magnification leading directly a pixel size of $0.39 \mathrm{~nm}$. Scale bar: $20 \mathrm{~nm}$. 

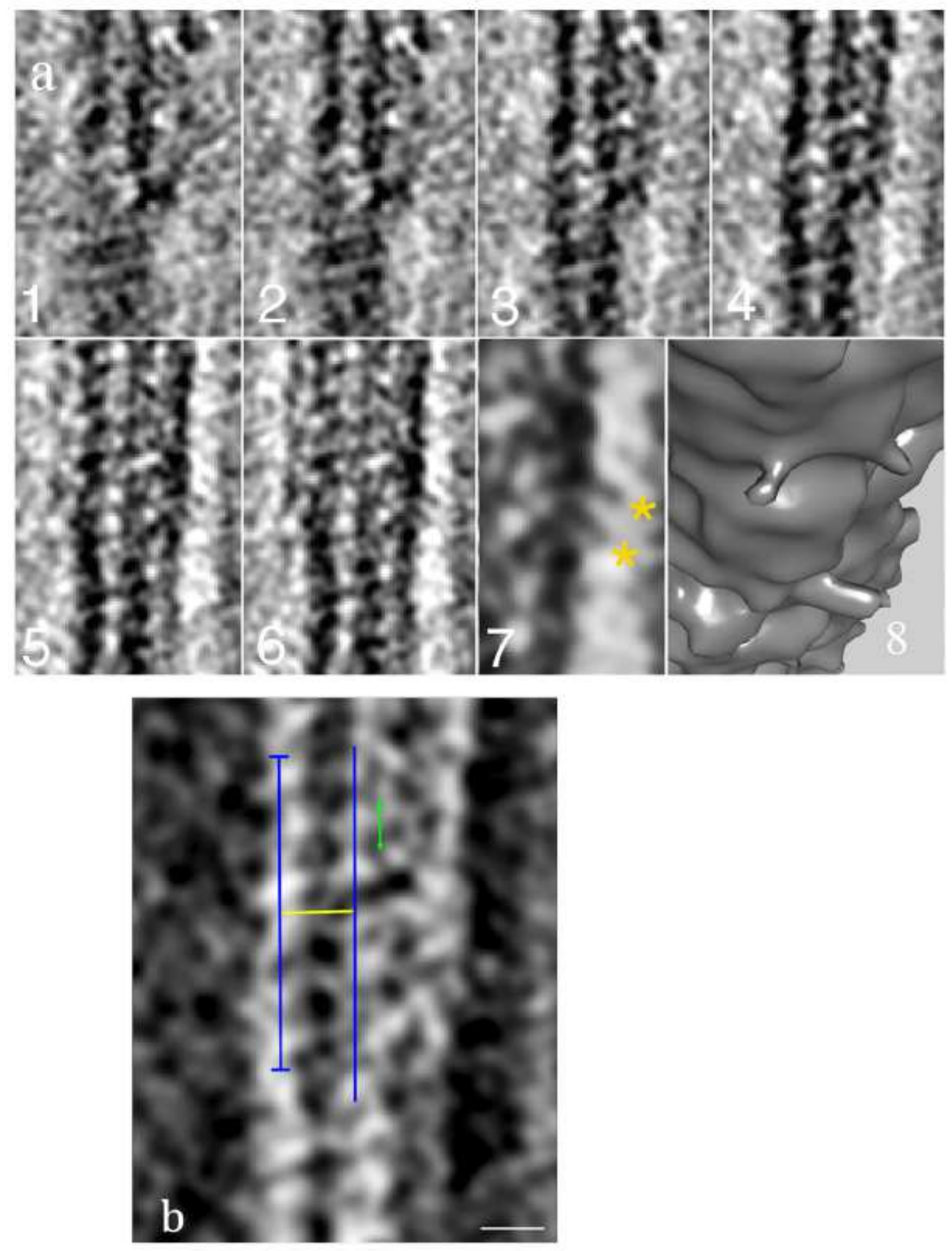

\section{Figure 2}

Virtual sections extracted from corresponding tomogram calculated from this tilt series. All images recorded between Fig 1A and Fig. 1B (cfr. text and supplementary material). Fig.2a: Details of virtual sections from tomogram, composed with enlarged virtual section (panel 7), and corresponding threedimensional rendering of a portion of the outer surface of this microtubule (panel 8). Contrast inverted as in Fig. 1: the stain appears lighter color in this picture. Voxel size is $0.39 \mathrm{~nm}$. The successive virtual 
sections show roughly half a microtubule, starting from its contact with the support film (panel 1) to its hollow center (panel 6). Individual subunits of protofilaments appear as non-spherical, globular structures whose long axis is at an angle to the long protofilament axis. Protrusions are seen emanating from each subunit, which do not appear all identical in shape (see discussion). Most evident CTT in panel 6 are reproduced, magnified and rescaled in panel 7, where CTT are indicated by yellow asterisks. Panel 8 is the three-dimensional rendering close-up of a portion of this microtubule, displaying at least a few CTTs. Rendering realized with Chimera (shareware from UCSF) choosing a uniform threshold after correcting the reconstruction for increasing scattering at decreasing surface angles (see methods section 1). Fig. 2b: Expanded virtual section extracted from same portion of tomogram (panel 4) after digitally rescaling of the pixels (Image J). Here contrast is not inverted (tubulin is brilliant while stain is darker) in order to facilitate comparison with colored lines. Blue lines indicate two protofilaments; a representative spacing of $\sim 5.7 \mathrm{~nm}$ is indicated by the yellow line. Double-headed green arrow line indicate monomer dimensions $(\sim 4 \mathrm{~nm})$.Scale bar: $5 \mathrm{~nm}$.

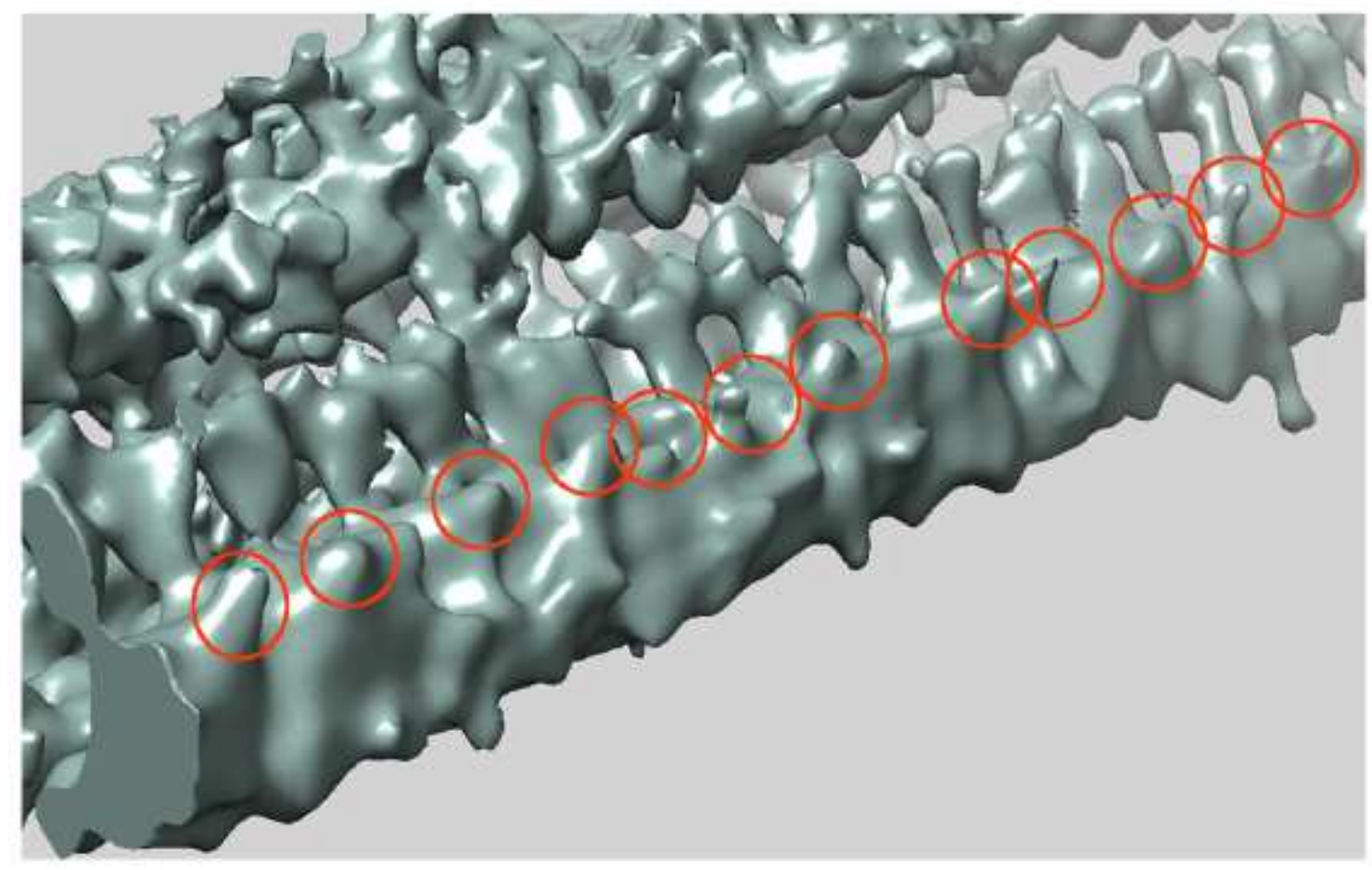

\section{Figure 3}

Rendering of the microtubule in Figure 2. Picture realized imposing a uniform threshold. Microtubule cross sectional width is $28.6 \mathrm{~nm}$ while height is $15.3 \mathrm{~nm}$. Regularly spaced stalks (some circled in red) are evident on each side of the microtubule. The statistical error of the reconstructed signal is lowest at the extreme lateral sides of microtubules (see methods), where these regular spikes are most apparent every $4.0 \pm 0.8(22) \mathrm{nm}$ on average. These features are mixed with noise at tops of microtubule. (See discussion). Uniform threshold in this picture has been carefully chosen so that all details shown are well above the signal-to-noise ratio. 


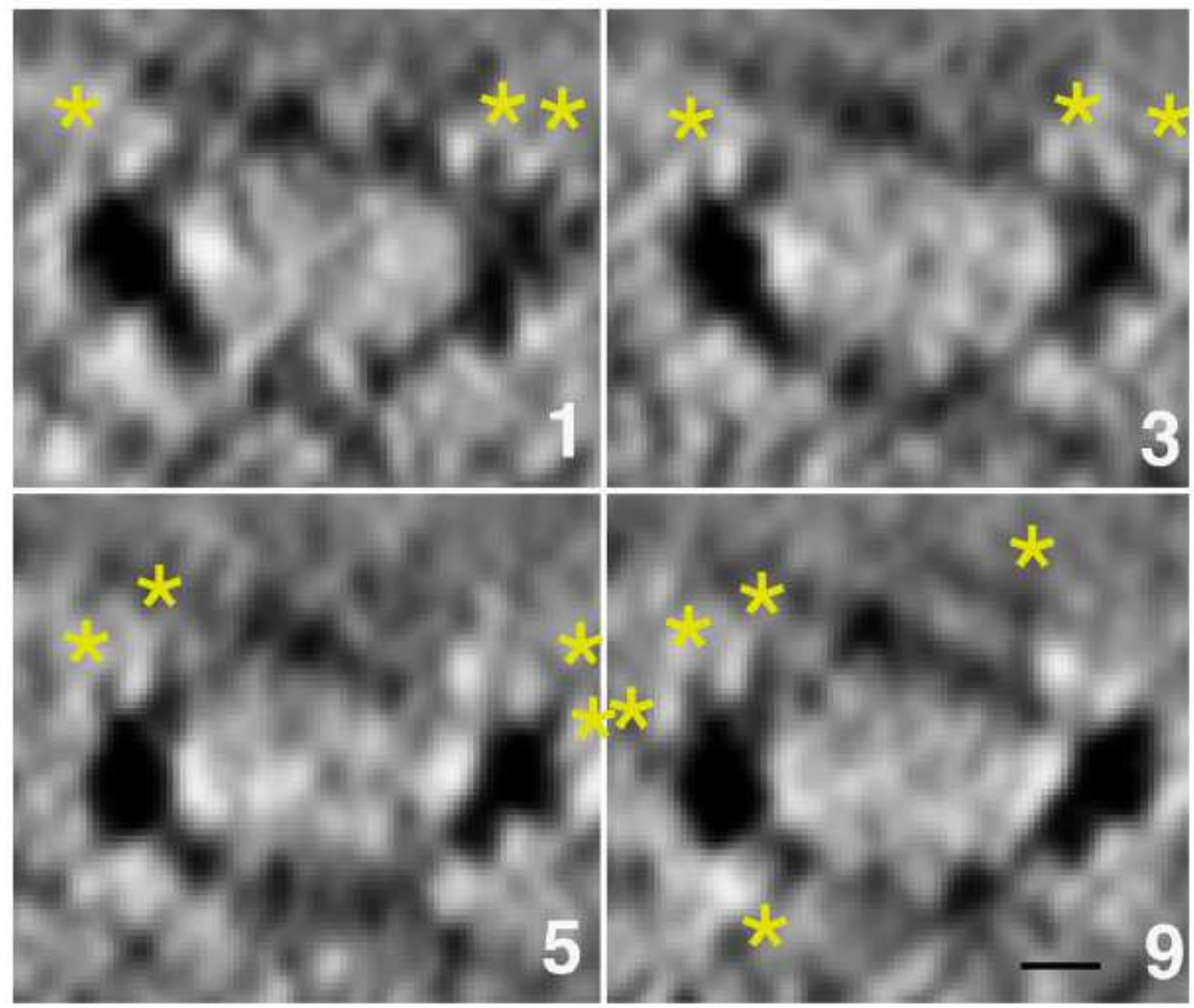

\section{Figure 4}

Virtual sections $\sim 0.39 \mathrm{~nm}$ thick in the $\mathrm{xz}$ plane, after aligning y with protofilament long axis. Contrast has been inverted. Stars indicate CTT resolved with high accuracy. Numbers reported at the right bottom corner indicate section position along the $y$-axis, corresponding to the long axis of the microtubule imaged here. Virtual sections are $0.39 \mathrm{~nm}$ thick. CTT appear and disappear again moving along the protofilaments. CTTs have a maximal thickness at half-length of $\sim 1 \mathrm{~nm}$ and a maximal length of $\sim 2.5$ $\mathrm{nm}$. Corresponding virtual sections of a microtubule digested with subtilisin (see text) prior to observation are reported in supplementary material. Scale bar: $5 \mathrm{~nm}$. 


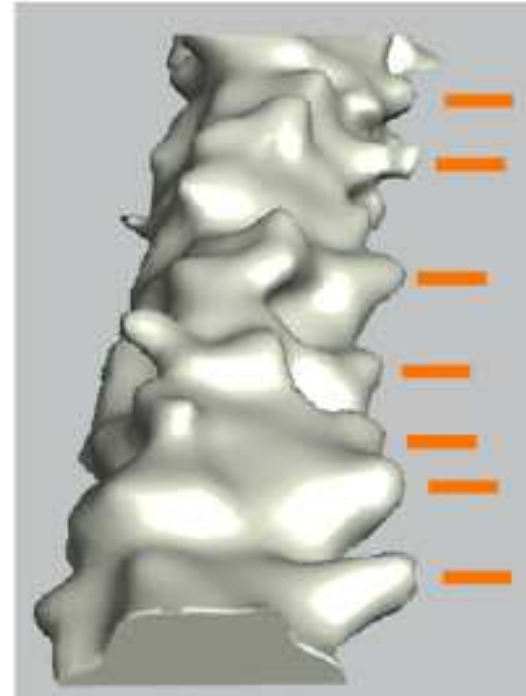

control

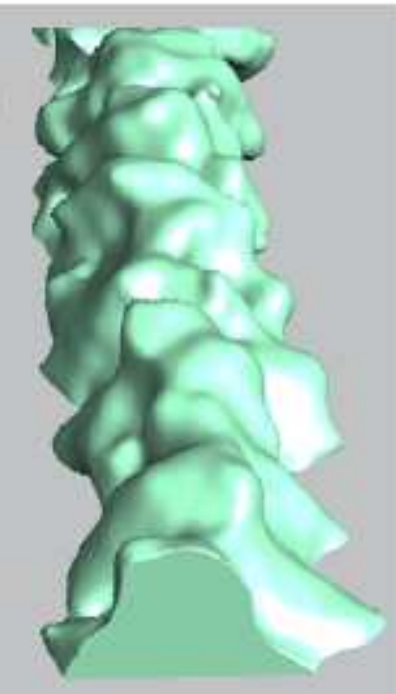

subtilisin

\section{Figure 5}

Three-dimensional rendering of the most lateral protofilament corresponding to two distinct microtubules, only one digested with subtilisin. Here $\mathrm{S} / \mathrm{N}$ is maximum and immediately comparable (see methods for discussion). The microtubule on the left is undigested, while the one on the right was digested with Subtilisin. C-terminal peptides are clearly visible only on the left rendering. Orange lines indicate some peptides on the undigested microtubule where they have an average period of $4.0 \pm 0.8 \mathrm{~nm}$ (22).

\section{Supplementary Files}

This is a list of supplementary files associated with this preprint. Click to download.

- S1.pdf

- S2.pdf

- S3.pdf

- MethodsandSupplementaryMaterial.pdf 